\title{
A quantum Monte Carlo study of electron correlation in transition metal oxygen molecules
}

\author{
Lucas Wagner *, Lubos Mitas \\ Department of Physics, North Carolina State University, Raleigh, NC 27695-8202, USA
}

Received 1 January 2003; in final form 1 January 2003

\begin{abstract}
We carry out calculations of selected transition metal-oxygen molecules within density functional theory, postHartree-Fock, and quantum Monte Carlo (QMC) methods. Transition metal-oxygen systems have competing electron correlation and exchange effects and require accurate treatment of both of these effects. We analyze the biases of the mentioned methods and their impacts on the electronic structure. We evaluate binding energies and compare the accuracy of various approaches including single and multi-reference trial wave functions in QMC.
\end{abstract}

(c) 2003 Elsevier Science B.V. All rights reserved.

\section{Introduction}

Bonding between oxygen and a transition metal is present in an astonishing variety of important molecular and solid systems, which include key biomolecular reaction centers, cluster catalysts, and various solid materials such as magnetic, ferroelectric, and superconducting systems. The exact nature of the bonding varies from system to system and results from combined effects of charge transfer, covalency, electronic exchange and correlation, and, for solid systems, crystal field impacts. Not surprisingly, these systems have been at the forefront of electronic structure research for several decades and will

\footnotetext{
${ }^{*}$ Corresponding author. Fax: 1-9195134496.

E-mail address: ikwagner@unity.ncsu.edu (L. Wagner).
}

remain in the center of interests for many years to come. Most studies have employed mainstream approaches such as Hartree-Fock and post-Hartree-Fock methods for smaller systems and/or density functional theory (DFT) approaches such as local spin density (LSDA) or variety of generalized gradient functionals (GGA). However, post-Hartree-Fock methods are limited to systems of small sizes and not really applicable to periodic systems. The DFT methods often lack accuracy and sometimes need ad hoc additional ingredients to provide the very elementary physics right such as is the case of LDA+U theories [1]. One can mention more examples, e.g., calculations of $\mathrm{BaTiO}_{3}$, a model perovskite ferroelectric, show significant errors in the optimal volume as predicted by DFT approaches [2] and require further elaboration in order to reproduce the experimental data. 
In this Letter we apply the quantum Monte Carlo (QMC) methods to transition metal-oxygen molecules in order to test the performance and accuracy of the method on this type of system in a simplest form. The QMC method has proved to be very successful in a number of previous applications to sp-systems such as predictions of energy ordering of $\mathrm{C}_{20}$ isomers, silicon clusters, chemical reactions, excited states in molecules and nanocrystals $[3 \mathrm{a}-3 \mathrm{~d}]$.

To the best of our knowledge, very little has been done for applying QMC methods to the transition metal systems, especially with the highaccuracy diffusion Monte Carlo method. One of us did a thorough study of excited states of Fe atom some time ago [4] which showed the accuracy of $0.1-0.2 \mathrm{eV}$ for excited states, followed by studies of $\mathrm{Hg}$ clusters [5]. Recently, Lüchow and Sokolova [6] estimated the singlet-triplet splitting for TiC molecule and obtained quite accurate results on par with the most extensive coupled cluster $\operatorname{CCSD}(\mathrm{T})$ results with extensive basis sets. We perform high-accuracy DMC calculations with explicit electron-electron correlation on model systems $\mathrm{MnO}$ and $\mathrm{TiO}$.

\section{Computational method}

The variational and diffusion Monte Carlo methods are described at length elsewhere [7] and therefore we briefly mention only the main points. The variational wave function is given by the product of the determinantal part and correlation

$\Psi_{T}=\operatorname{Det}\left[\phi_{1}, \phi_{2}, \ldots, \phi_{n}\right] \operatorname{Det}\left[\phi_{1}, \phi_{2}, \ldots, \phi_{m}\right] e^{U}$,

where

$U=\sum_{i<j, I} u\left(r_{i I}, r_{j I}, r_{i j}\right)$,

is a function of the electron-electron $\left(r_{i j}\right)$ and electron-ion $\left(r_{i I}, r_{j I}\right)$ distances and includes the exact cusp conditions between the like and unlike pairs of electrons. The energy is evaluated by Monte Carlo integration using samples of electronic positions distributed according to $\Psi_{T}^{2}(\mathbf{R})$, where $\mathbf{R}=\left(\mathbf{r}_{1}, \mathbf{r}_{2}, \ldots, \mathbf{r}_{n}\right)$. The variational energy is given by
$E_{\mathrm{VMC}}=\frac{1}{M} \sum_{m} \frac{H \Psi_{T}(\mathbf{R})}{\Psi_{T}(\mathbf{R})}+\mathcal{O}(1 / \sqrt{M})$,

where $M$ is the number of samples and $H$ is the electron-ion Hamiltonian in the Born-Oppenheimer framework.

The correlation factor typically includes about 30 variational parameters which are optimized by minimization of the energy variance

$\sigma^{2}=(1 / M) \sum_{m}\left(E_{L}\left(\mathbf{R}_{m}\right)-E_{\mathrm{VMC}}\right)^{2}$,

where $E_{L}=H \Psi_{T}\left(\mathbf{R}_{m}\right) / \Psi_{T}\left(\mathbf{R}_{m}\right)$. The variational wavefunctions typically account for about $85 \%$ of the correlation energy. The optimized wavefunctions are then used as important sampling distributions for the diffusion Monte Carlo (DMC) method. In the diffusion Monte Carlo method one applies the propagator $e^{-\tau H}$ to an initial wavefunction, typically, $\Psi_{T}$. This projection is most conveniently carried out by stochastically solving the Schrödinger equation in imaginary time

$f_{\mathrm{DMC}}(\mathbf{R}, t+\tau)=\int G\left(\mathbf{R}, \mathbf{R}^{\prime}, \tau\right) f_{\mathrm{DMC}}(\mathbf{R}, t) \mathrm{d} \mathbf{R}^{\prime}$,

where

$G\left(\mathbf{R}, \mathbf{R}^{\prime}, \tau\right)=\Psi_{T}(\mathbf{R}) \Psi_{T}^{-1}\left(\mathbf{R}^{\prime}\right)\left\langle\mathbf{R}|\exp (-\tau H)| \mathbf{R}^{\prime}\right\rangle$.

It is straightforward to verify that in the limit of the imaginary time $\tau \rightarrow \infty, f_{\mathrm{DMC}}=\Psi_{T} \Psi_{0}$ where $\Psi_{0}$ is the ground state which has a nonzero overlap with $\Psi_{T}$. The fermion sign problem is circumvented by requiring the nodes of $\Psi_{0}$, the DMC solution to be identical to the nodes of $\Psi_{T}$, so we enforce the so-called fixed-node approximation. This introduces about 5\% error in the correlation energy, but due to partial error cancellation, the method is able to get energy differences typically within $1-2 \%$ of experiment [10].

In our calculations, the core electrons are replaced by the effective core potentials. For Ti and Mn we use recently generated ECPs from DiracFock calculations [8] with Ne-core, thus including the $3 \mathrm{~s}$ and $3 \mathrm{p}$ states into the valence space. For oxygen, we use recently generated soft-core ECPs [9]. The gaussian basis sets we used were adjusted to these ECPs and were contracted as (12s10p9d1f)/ 
[6s4p4d1f] for Ti, (12s12p7d1f)/[6s6p3d1f] for Mn and $(9 \mathrm{~s} 9 \mathrm{p} 1 \mathrm{~d}) /[3 \mathrm{~s} 3 \mathrm{p} 1 \mathrm{~d}]$ for $\mathrm{O}$.

Our main focus was to establish the levels of accuracy that can be obtained from single vs. multi-reference wavefunctions and to test sets of one-particle orbitals form Hartree-Fock, multiconfigurations self-consistent field (MCSCF) and also DFT methods. We also wanted to compare the results with some of the GGA/DFT methods, which are often used in large scale calculations. We therefore compare the following trial wavefunctions: single reference Hartree-Fock, single reference with orbitals from the B3LYP functional and multi-reference wavefunctions with MCSCF. Besides the calculations of the $\mathrm{TiO}$ and $\mathrm{MnO}$ molecules, we also carried out $\mathrm{s} \rightarrow \mathrm{d}$ excitation energy calculations of the $\mathrm{Mn}$ atom. These are well known to be difficult especially for the elements on the middle of the transition row. We used the packages Gamess [10] and Gaussian [11] for the non QMC methods.

\subsection{Hartree-Fock single reference}

We first tried using HF orbitals in a single reference trial functions for QMC. From all the types of the wavefunctions we used, this turned out to be the least accurate one. This was rather unexpected since our previous calculations in sp-systems usually resulted in marginal differences between HF and DFT orbitals (admittedly the number of carried out tests was quite limited). While the HF orbitals provided excellent estimation of $3 d^{5} 4 s^{2} \rightarrow 3 d^{6} 4 s^{1}$ transition energy, we find that it shows the largest discrepancies with experiment in the atomization energy of the dimers. The poor performance of Hartree-Fock orbitals suggests that correlation is important already at the level of an effective one-particle theory and has nontrivial effects on the nodes of the trial wave functions

\subsection{B3LYP single reference}

The B3LYP functional, originally proposed by Becke [12-14], includes a fraction of the exact exchange; therefore, compared to other DFT techniques, it is better able to describe systems with large exchange, such as transition metals. While

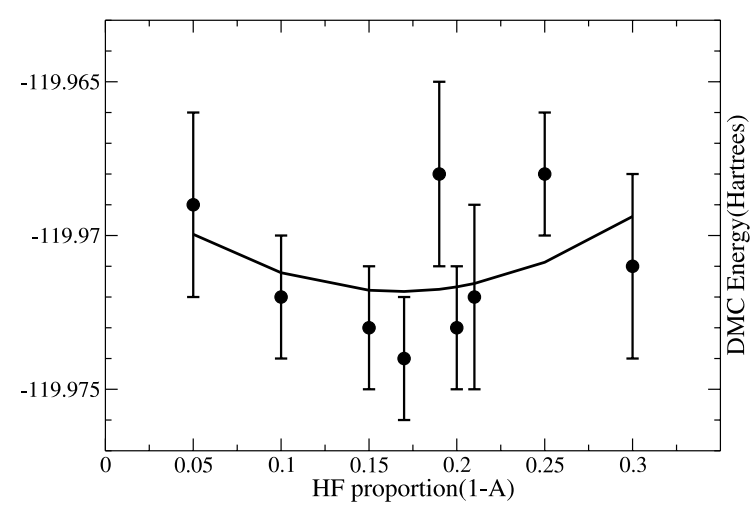

Fig. 1. MnO energy as a function of the weight of exact exchange in the B3LYP-type of GGA functional (see text). The solid line is a quadratic fit to DMC data with plotted error bars.

the DFT orbitals were originally introduced as a convenient way to express the one-electron density, they provide an interesting opportunity to test the performance of the B3LYP functional as an effective one-particle theory. Since a corresponding effective Hamiltonian tunes the 'balance' between exchange and correlation it is clear that it can have impact on quality of orbitals in transition metal systems where both exchange and correlation are large. We also attempted to improve the virtual B3LYP orbitals by reoptimizing them in a subsequent MCSCF calculation, however, there was no noticeable improvement.

We also attempted to adjust the proportion of exact exchange in the B3LYP functional. The $\mathrm{B} 3 \mathrm{LYP}$ functional has the form $E_{\mathrm{B} 3 \mathrm{LYP}}=A E x_{\text {Slater }}+$ $(1-A) E x_{\mathrm{HF}}+B D E x_{\text {Becke }}+E c_{\mathrm{LDA}}+C D E c_{\mathrm{LYP}} . \mathrm{We}$ varied the $A$ parameter, keeping the rest constant. It has been shown [15] that $(1-A)=0.15$ gives better agreement for transition metal oxides. For the DMC energy, the optimal proportion for $\mathrm{MnO}$ appears to be approximately 0.17 (Fig. 1). However, in this case, the energy changes from using a modified B3LYP functional are quite small with marginal impact on the energy differences.

\section{3. $M C S C F$}

In our MCSCF calculations, we froze the transition metal $3 \mathrm{~s}$ and $3 \mathrm{p}$ states and used single and double excitations to obtain a higher quality starting trial wavefunction at the determinantal 
Table 1

DMC total energies of $\mathrm{MnO}$ with multi-reference wavefunctions with respect to active space in MCSCF calculation (units are Hartrees)

\begin{tabular}{llll}
\hline $\begin{array}{l}\text { Active } \\
\text { space }\end{array}$ & $\begin{array}{l}\text { MCSCF } \\
\text { energy }\end{array}$ & $\begin{array}{l}\text { DMC } \\
\text { energy }\end{array}$ & $\begin{array}{l}\text { DMC } \\
\text { determinants }\end{array}$ \\
\hline 10 & -119.269 & $-119.974(4)$ & 27 \\
12 & -119.296 & $-119.973(4)$ & 18 \\
17 & -119.343 & $-119.967(4)$ & 21 \\
\hline
\end{tabular}

level. For $\mathrm{MnO}$, we added also an atomic natural orbital in the $\mathrm{f}$ channel of $\mathrm{Mn}$ and in the $\mathrm{d}$ channel of oxygen. These orbitals were similar to the ones used in all-electron calculations, except that they were smoothed in the deep core region to adjust for the effective core potential in that region. We used the MCSCF orbitals in a CI calculation, and used determinants with weights above 0.05 for $\mathrm{MnO}$ and 0.01 for TiO. These thresholds kept the number of determinants close to 20 . For TiO, we used an active space of 16 orbitals, and had 23 determinants in the QMC calculation. For $\mathrm{MnO}$, we tried active spaces of 10,12 , and 17 orbitals. Increasing the active space had only marginal effect on improvements of the wavefunctions, at least within the limits of our investigations. In fact, having a larger active space seems to do very little to improve the nodes in this case, so we used the small active space for the binding energy calculations (Table 1). Since our trial functions contain only a subset of configuration state functions from the CI expansion it is quite likely that the energy convergence with respect to the active space is not always monotonic.

\section{Results}

As we mentioned before, the $\mathrm{s} \rightarrow \mathrm{d}$ excitations in $3 \mathrm{~d}$ atoms are quite challenging and require recovery of large amount of dynamical correlation in post-Hartree-Fock calculations. The difficulty is in the double occupation of the d-shell, which creates large electron-electron repulsion with significant increase in the importance of correlation. As an example we calculated the promotion energy from $\mathrm{d}^{5} \mathrm{~s}^{2} \rightarrow \mathrm{d}^{6} \mathrm{~s}$ for the manganese atom. Indeed, the DFT results show significant differences when compared with experiment. Note that B3LYP is still off by almost $0.4 \mathrm{eV}$ by not having the 'optimal' balance between the exchange and correlation. It is also clear that for a single atom the fixed-node errors of single-reference wavefunction largely cancel out in DMC differences and the results are very close to the experiment (see Table 2). Since both HF and B3LYP orbitals gave essentially the same energies, the choice of orbitals was less important in this case, indicating that both orbital sets resulted in wave functions with similar nodal surfaces. Note that the DMC methods provide a large improvement when compared with the rest of the methods.

While it was rather straightforward to find the bond length for the TiO molecule, we found that for $\mathrm{MnO}$ there was some discrepancy between various sources on the equilibrium bond length. An older reference [17] gives the bond length as $1.769 \AA$, which is very close to the Hartree-Fock optimized bond length. However, a newer reference [18] gives the bond length as $1.648 \AA$, which is very similar to the DFT and QMC methods. Since the DFT and QMC results agree very reasonably we predict that the smaller value is closer to the actual bond length (see Tables 3 and 4).

Table 2

$\mathrm{Mn}$ atom $\mathrm{s} \rightarrow \mathrm{d}$ promotion energies by various methods

\begin{tabular}{ll}
\hline & $\begin{array}{l}\mathrm{d}^{5} \mathrm{~s}^{2} \rightarrow \mathrm{d}^{6} \mathrm{~s} \\
(\mathrm{eV})\end{array}$ \\
\hline HF & 3.5 \\
LSDA/SVWN5 & 1.38 \\
GGA/BLYP & 1.22 \\
GGA/B3LYP & 1.65 \\
DMC(HF orbitals) & $2.2(1)$ \\
DMC(B3LYP orbitals) & $2.1(1)$ \\
Experiment & 2.0 \\
\hline
\end{tabular}

Table 3

Minimum energy bond lengths $(\AA)$

\begin{tabular}{ll}
\hline & $\mathrm{MnO}$ \\
\hline Hartree-Fock & $1.734[16]$ \\
LSDA/SVWN5 & 1.625 \\
GGA/PW91 & 1.625 \\
GGA/B3LYP & 1.625 \\
DMC(HF orbitals) & $1.65(5)$ \\
Experiment & $1.769[17]$ or $1.648[18]$ \\
\hline
\end{tabular}


Table 4

Binding energies $(\mathrm{eV})$ by various methods

\begin{tabular}{lll}
\hline & $\mathrm{TiO}$ & $\mathrm{MnO}$ \\
\hline State & ${ }^{3} \Delta$ & ${ }^{6} \Sigma$ \\
Hartree-Fock & $2.64[16]$ & $-0.92[16]$ \\
LSDA & 9.12 & 5.88 \\
PW91 & 7.45 & 4.79 \\
B3LYP & 6.62 & 3.39 \\
VMC(HF orbitals) & $6.0(1)$ & $3.1(1)$ \\
DMC(HF orbitals) & $6.3(1)$ & $2.9(1)$ \\
DMC(B3LYP orbitals) & $6.9(1)$ & $3.4(2)$ \\
DMC(MCSCF orbitals) & $6.7(2)$ & $3.4(2)$ \\
Experiment & $6.98[17]$ or & $3.70[17]$ \\
& $6.87(7)[19]$ & \\
\hline
\end{tabular}

The DMC(MCSCF) was a multi-reference wave function with $\approx 20$ configurations with the largest weights.

The binding energy showed typical patterns of the HF vs. DFT methods, with the DFT approaches overbinding, and HF underbinding. B3LYP obtains very reasonable results, and as we improve the trial wavefunctions, we can see the DMC binding energy converging to the experimental one.

\section{Discussion}

It is interesting to compare the performance of various methods. For example, it is clear that PW91, which works very reasonably for sp-systems, is less predictive in systems with d-elements leading to errors of almost $1 \mathrm{eV}$ (observed also for excitations in $\mathrm{Fe}$ [4]).

Using only a single-reference Hartree-Fock trial function, we underestimate the binding energy of $\mathrm{TiO}$ by about $0.6 \mathrm{eV}$, and $\mathrm{MnO}$ by $0.8 \mathrm{eV}$. We can eliminate about one half of this error by improving the trial nodal surface with multiple determinants in a MCSCF calculation. Using B3LYP orbitals improved the calculated binding energy by a surprisingly large amount, even surpassing the performance of the MCSCF wavefunction in TiO. The result which calls for further investigation is the slow and oscillatory improvement from increasing the size of the MCSF wavefunctions as has been observed also by others $[6,20]$. Since using CI or MCSCF is not computationally feasible for large systems and solids, B3LYP may offer a way to obtain improved quality wavefunctions for TMO systems.
It is clear that the increase of the number of d-electrons in the system makes the calculations more challenging. Since $\mathrm{MnO}$ has three more d-electrons, the correlations are larger and the discrepancies with experiment are more pronounced at the Hartree-Fock level despite the fact that the system is highly spin-polarized. The impact of the correlation on the bond length of $\mathrm{MnO}$ is quite clear as well.

In conclusion, we have compared several different methods in calculating the binding energy of $\mathrm{MnO}$ and $\mathrm{TiO}$, and an excitation of $\mathrm{Mn}$. The DMC methods perform very well, and the agreement with experiment is encouraging. The strong impact of the choice of orbitals is interesting and calls for further investigations. The fact that B3LYP orbitals do lead to wavefunctions with improved nodes signals that it might be worthwhile to look into the improvements of the effective one-particle theories to produce the orbitals. One way to accomplish this was suggested by Filippi and Fahy [21]. The fixed-node approximation is visible in the binding energy of $\mathrm{MnO}$; nevertheless, the results are very promising considering the fact that we did not exhaust all the possibilities to improve the wavefunction such as reoptimizing the orbitals in the presence of Jastrow correlation factors and more extensive employment of the MCSCF method. The results provide an interesting benchmark for these types of systems and enabled us to estimate and predict the equilibrium bond length for the $\mathrm{MnO}$ molecule.

\section{Acknowledgements}

Support by ONR-N0014-01-1-0408, DARPA/ ONR-N00014-01-1-1062 and NSF-DMR-0102668 grants is gratefully acknowledged. We are also grateful to Dr. Y. Lee for providing us with his recently developed Dirac-Fock ECPs for the $3 \mathrm{~d}$ atom series.

\section{References}

[1] See, for example, M.A. Korotin, A.V. Postnikov, T. Neumann, G. Borstel, V.I. Anisimov, M. Methfessel, Phys. Rev. B 49 (1994) 6548, and references therein. 
[2] S. Tinte, M.G. Stachiotti, C.O. Rodriguez, D.L. Novikov, N.E. Christensen, Phys. Rev. B 58 (1998) 11959.

[3] (a) J.C. Grossman, L. Mitas, Phys. Rev. Lett. 74 (1995) 1323 ;

(b) J.C. Grossman, L. Mitas, K. Raghavachari, Phys. Rev. Lett. 75 (1995) 3870;

(c) J.C. Grossman, L. Mitas, Phys. Rev. Lett 79 (1997) 4353;

(d) J.C. Grossman, M. Rohlfing, L. Mitas, S.G. Louie, M.L. Cohen, Phys. Rev. Lett. 86 (2001) 472.

[4] L. Mitas, Phys. Rev. A 49 (1994) 4411.

[5] M. Dolg, H.J. Flad, Mol. Phys. 91 (1997) 815.

[6] S. Sokolova, A. Lüchow, Chem. Phys. Lett. 320 (2000) 421.

[7] W.M.C. Foulkes, L. Mitas, R.J. Needs, G. Rajagopal, Rev. Mod. Phys. 73 (2001) 1.

[8] Y. Lee, P.R.C. Kent, M.D. Towler, R.J. Needs, G. Rajagopal, Phys. Rev. B 62 (2000) 13347, and private communication.

[9] I. Ovcharenko, A. Aspuru-Guzik, W.A. Lester Jr., J. Chem. Phys. 114 (2001) 7790.

[10] U.S. Gamess, M.W. Schmidt, K.K. Baldridge, J.A. Boatz, S.T. Elbert, M.S. Gordon, J.H. Jensen, S. Koseki, N.
Matsunaga, K.A. Nguyen, S.J. Su, T.L. Windus, M. Dupuis, J.A. Montgomery, J. Comput. Chem. 14 (1993) 1347.

[11] M.J. Frisch et al., Gaussian 98, Revision A.11.4, Gaussian, Inc., Pittsburgh PA, 2002.

[12] A.D. Becke, Phys. Rev. A 38 (1988) 3098.

[13] C. Lee, W. Yang, R.G. Parr, Phys. Rev. B 37 (1988) 785.

[14] A.D. Becke, J. Chem. Phys. 98 (1993) 5648.

[15] O. Salomon, M. Reiher, B.A. Hess, J. Chem. Phys. 117 (2002) 4729.

[16] M. Dolg, U. Wedig, H. Stoll, H. Preuss, J. Chem. Phys. 86 (1986) 2123

[17] K.P. Huber, G. Herzberg, Molecular Spectra and Molecular Structure. IV. Constants of Diatomic Molecules, Van Nostrand, New York, 1979.

[18] R.M Gordon, A.J. Merer, Can. J. Phys. 58 (1980) 642.

[19] H.P. Loock, B. Simard, S. Wallin, C. Linton, J. Chem. Phys. 109 (1998) 8980.

[20] Y. Shlyakhter, S. Sokolova, A. Lüchow, J.B. Anderson, J. Chem. Phys. 110 (1999) 10725.

[21] C. Filippi, S. Fahy, J. Chem. Phys. 112 (2000) 3523. 